\title{
Associations between family structure and young people's physical activity and screen time behaviors
}

\author{
Amund Langøy ${ }^{1}$, Otto R. F. Smith², Bente Wold ${ }^{3}$, Oddrun Samdal ${ }^{3}$ and Ellen M. Haug ${ }^{1,3^{*}}$
}

\begin{abstract}
Background: Identifying factors that can influence young peoples' physical activity and sedentary behaviors is important for the development of effective interventions. The family structure in which children grow up may be one such factor. As the prevalence of single parent and reconstituted families have increased substantially over the last decades, the objective of this study was to examine whether these family structures are differentially associated with young people's MVPA, participation in organized sports and screen-time activities (screen-based passive entertainment, gaming, other screen-based activities) as compared to traditional nuclear families.

Methods: The data stem from the 2013/2014 "Health Behaviour in School- aged Children (HBSC) study". A large Norwegian sample of 11-16years old students $(n=4509)$ participated. Cluster-adjusted regression models were estimated using full information maximum likelihood with robust standard errors (MLR).
\end{abstract}

Results: After adjusting for covariates, living with a single parent was negatively associated with days/week with 60 min MVPA ( $b=-.39,95 \% \mathrm{Cl}:-.58,-.20)$, and positively associated with hours/weekday of total screen time $(b=.50$, $95 \% \mathrm{Cl}: .08, .93)$. Young people living with a single parent were also more likely to report no participation in organized sports ( $\mathrm{OR}=1.40,95 \% \mathrm{Cl}$ : 1.09, 1.79). Living in a reconstituted family was negatively associated with days/ week with 60 min MVPA ( $b=-.31,95 \% \mathrm{Cl}:-.53,-.08)$, and positively associated with hours/weekday of total screen time $(b=.85,95 \% \mathrm{Cl}: 37,1.33)$. For all outcomes, the interaction effects of family structure with sex, and with having siblings were not statistically significant. For material affluence, a significant interaction effect was found for participation in organized sports $\left(x^{2}[4]=13.9, p=.008\right.$ ). Those living in a reconstituted family with low or high material affluence had an increased risk for not participating in organized sports whereas those with medium material affluence did not.

Conclusion: This study suggests that living with a single parent or in reconstituted families was unfavorably associated with physical activity, sport participation and screen-based behaviors among Norwegian youth. The findings indicate that family structure could be an important factor to take into account in the development and testing of interventions. More in-depth research is needed to identify the mechanisms involved.

Keywords: Family structure, Young people - physical activity, Sport participation, Screen time behaviors

\footnotetext{
* Correspondence: Ellen.haug@uib.no

${ }^{1}$ NLA University College, Bergen, Pb 74 Sandviken, 5812 Bergen, Norway

${ }^{3}$ Department of Health Promotion and Development, University of Bergen,

Christiesgate 13, 5020 Bergen, Norway

Full list of author information is available at the end of the article
}

(c) The Author(s). 2019 Open Access This article is distributed under the terms of the Creative Commons Attribution 4.0 International License (http://creativecommons.org/licenses/by/4.0/), which permits unrestricted use, distribution, and reproduction in any medium, provided you give appropriate credit to the original author(s) and the source, provide a link to the Creative Commons license, and indicate if changes were made. The Creative Commons Public Domain Dedication waiver (http://creativecommons.org/publicdomain/zero/1.0/) applies to the data made available in this article, unless otherwise stated. 


\section{Background}

Physical activity is associated with physiological [1, 2], psychological and social [3] health benefits for children and youth. A disconcerting trend with lower numbers fulfilling the recommendations of $60 \mathrm{~min} /$ day of moderate to vigorous physical activity (MVPA) is observed, with an overall age-related decline during adolescent years [4]. Simultaneously, young people's time spent on sedentary activities is increasing [4-6] and such activities may dominate their lives today [7]. Computer use for gaming and non-gaming purposes is sharply increasing and seems to counterweight the observed decrease in TV viewing [4]. Sedentary time and screen-based activities are negatively associated with young people's physical health, other adverse health behaviors and socio-cognitive outcomes $[8,9]$. Several countries have developed guidelines, often recommending that the after-school period should be limited to two hours per day with screen-based activities [10-13].

According to the behavioral epidemiology framework, factors that correlate with young peoples' physical activity and sedentary time need to be identified to inform the development of effective intervention strategies [14]. Based on a comprehensive review, Biddle et al. (2011) found parental influences to be the key issue regarding social and cultural correlates of physical activity in children. In particular, the existing literature suggests that parental support, either directly or indirectly affects children's physical activity level in three important ways; encouragement, involvement and facilitation, including financial resources $[15,16]$. There is a paucity of research when it comes to the relationship between parental influences and young people's sedentary behavior, and so far, only parental TV viewing appears to be a consistent correlate [8]. Nonetheless, results from interventions designed to reduce sedentary behavior suggest that the involvement of family and parents was an important ingredient for success [17].

The extent to which parental influences exert a positive or negative effect on young children's level of physical activity and sedentary behaviors, may also depend on the family structure in which children grow up. Children and youth are increasingly living in various types of family structures. In the EU the numbers of divorces [18], and the number of children being born of single parents, are growing [19]. Similar patterns are present in countries outside the EU, such as Australia [20], Canada [21] and China [22]. Research on family structure has increased dramatically the past two decades, drawing a complicated picture of family conditions and processes that are associated with healthy child development. In general, the literatures suggests that living with single parents or in reconstituted families are less favorable in both the cognitive and emotional-behavioral domains
[23], also in the context of the generous Nordic welfare states [24]. Simplified, the two primary mechanisms that might account for differences among families are time and money [24]. A higher likelihood of reduced economic resources in single-parent and reconstituted households has been documented [24-26]. Studies also suggest that children may receive less parental time and attention in both single-parent families and stepfamilies $[24,27]$.

The studies that have examined whether family structure is associated with young peoples' physical activity level [28-30], participation in sport [31-35], and screen time use [20,36,37], have so far resulted in inconclusive findings $[20,38,39]$. One major limitation is that family structure have almost exclusively been defined as simply single- or dual-parent, thereby ignoring the potential differences between traditional dual-parent families and reconstituted families that include a step-parent or a parent's partner [16].

Correlates for physical activity and sedentary behaviors are likely to be behavior specific. Organized sports is one of the most popular activities among young people $[40,41]$ and can contribute to a significant amount of young people's total physical activity [42, 43]. Few studies have identified correlates of sedentary activities with an explicit attention to the after-school period [44], and those that exist have mainly focused on TV viewing [8]. The objective of this paper was therefore to examine the association between various family structures (living with both parents, a single parent or in a reconstituted family) and young people's MVPA, organized sport participation and different types of after-school screen time use, including a reference to the existing guidelines.

\section{Methods \\ Participants}

The data was collected as a part of the 2013/2014 survey of the "Health Behaviour in School- aged Children (HBSC) study: A WHO collaborative national study". This cross-sectional survey had school class as the primary sampling unit, the classes were chosen from a geographically stratified list, to ensure a nationally representative sample. A sample of 11-, 13-,15- and 16 years old students $(n=4509)$ participated, with a class level response rate of $21 \%$ and a student level response rate of $76 \%$. At a school/class level a high work load and frequent requests regarding survey participation was reported as the main reasons for non-response. Absence on the day the survey was conducted, was the most frequent cause of non-response at the student level. The Norwegian Western Regional Ethical Committee (REK) approved the study and the use of passive consent. A detailed information letter was given both in paper form and electronically to parents or custodians for all 
participants below the age of 16 . Those that did not want their child to participate had to sign and return a form to the teacher. Approval of the child's participation was assumed if the form was not returned. The class teachers administered the survey. Participation was voluntary, and the anonymity as well as the confidentiality of the participants were ensured. More details on the HBSC study procedures can be found elsewhere [45].

\section{Measures}

Family structure was measured with one item "Please answer this first question for the home where you live all or most of the time and tick the people who live there". The response categories were: Mother, father, stepmother (or father's partner), stepfather (or mother's partner) and others (e.g. living with grandparents). The data was coded into four categories (both parents, single parent, reconstituted families and others). Participants in the 'others' category $(1.8 \%, n=83)$ were excluded from the analyses.

Moderate to vigorous physical activity (MVPA) was presented to the participants with the following statement: With physical activity we mean any activity that increases your heart rate and makes you get out of breath some of the time. Physical activity can be done in sports, school activities, playing with friends, or walking to school. Some examples of physical activity are running, biking, dancing, skateboarding, swimming, soccer, skiing/ snowboarding. For the next question, please summarize all the time you were physically active for every day. This description was followed by the item "Over the past 7 days, on how many days were you physically active for a total of at least 60 minutes per day?" There were eight response categories ( 0 days, 1 day up to 7 days). The item has been proven to have reasonable validity and moderate reliability [46] and acceptable correlation with accelerometer measures $[47,48]$. To reflect the recommended 60 min MVPA a day [49], a dichotomized variable with 7 days $=1$ and other responses $=0$ was computed.

Organized sport was measured by the combination of "How often do you usually participate in organized team sports?" and How often do you usually participate in organized individual sports? These items had four categories (Not at all, 2-3 times a month, once a week, twice a week or more). The items were dichotomized with those answering 'Not at all' on both items $=1$, other combinations $=0$. The items have shown acceptable levels of agreement, indicating good reliability [50].

Screen-based behaviors were assessed by three items: "How many hours a day, in your free time, do you usually spend watching TV, videos (including YouTube or similar services), DVDs, and other entertainment on a screen?", "How many hours a day, in your free time, do you usually spend playing games on a computer, games console, tablet (like iPad), smartphone or other electronic device (not including moving or fitness games)?" and "How many hours a day, in your free time, do you usually spend using electronic devices such computers, tablets (like iPad) or smart phones for other purposes, for example, homework, emailing, tweeting, Facebook, chatting, surfing the internet?". All items had 9 categories $(0,0.5,1$ to more than $7 \mathrm{~h})$. Participants answered these questions separately for weekdays and weekends. The items have had acceptable test-retest correlations [51-54]. A sum score of the three items measuring total hours a day with screen time was computed, and a cutoff at $2 \mathrm{~h} /$ day was set to extract an additional dichotomized variable to reflect recommendations.

Previous research has shown associations of young people's physical activity and screen-based behavior with gender, age, material affluence (SES), having siblings and BMI [31-33, 37, 55-59], and these variables were controlled for.

Material affluence was measured by a summary score of The Family Affluence Scale III (FAS-III). The FAS consists of the following six items: Does your family own a car, van or truck? (Responses: no, one, two or more); Do you have your own bedroom for yourself? (No, yes); How many times did you and your family travel out of Norway for a holiday/vacation last year? (Not at all, once, twice, more than twice); How many computers do your family own? (None, one, two, more than two); Does your family have a dishwasher at home? (No, yes); and How many bathrooms (rooms with a bath/shower or both) are in your home? (None, one, two, more than two) $[45,60]$. These six items provide a scale that can be easily completed by adolescents. A ridit transformation (conversion to cumulative probabilities) by age and sex was carried out on the sum score of the FAS-III items. The ridit scores were subsequently categorized into three groups with varying levels of relative material affluence: low (lowest 20\%), medium (between 20th and 80th percentile) and high (highest 20\%) [60].

Body mass index (BMI) using the formula $\mathrm{kg} / \mathrm{m} 2$ was assessed by two items: "How much do you weigh without clothes?" (in $\mathrm{kg}$ ) and "How tall are you without shoes?" (in $\mathrm{cm}$ ). The BMI scores were recoded into standardized z-scores based upon the guidelines from Childhood Obesity Working Group of the International Obesity Taskforce [61]. Self-reported BMI tends to underestimate the prevalence of obesity [62-66], and the associations of self-reported BMI with other variables are biased when BMI is used as a categorical variable [67]. The latter bias tends to be much lower when BMI is used as a continuous variable [65,67].

Siblings was measured by two items referring to where the respondent lived all or most of the time: "Please indicate how many brothers and sisters live here (including 
half, step or foster brothers and sisters)" (How many brothers?, How many sisters?).

\section{Statistical analyses}

SPSS 23.0 was used to calculate descriptive statistics. Mplus 7.4 was used for all other analyses. Due to the clustered sampling design, the "complex" option in Mplus was used to account for dependency between observations from the same school class. Substantial missing data was present for variables; screen-based passive entertainment (17.0\%), gaming (19.0\%), other screen-based activities (19.7\%), parental status (11.4\%), BMI (12.9\%), participation in organized PA (12.4\%), and having siblings (10.9\%). Full Information Maximum Likelihood (FIML) with robust standard errors (MLR) was adopted to deal with missing data and non-normality. FIML is valid under the assumption of missing at random, which is generally considered to be a more realistic assumption as compared to missing completely at random. MVPA (8-point scale), screen-based passive entertainment, gaming, other screen-based activities and total screen time (9-point scales) were treated as continuous dependent variables. Although formal normal distribution and homoscedasticity assumptions were not met for these variables, MLR estimation is known to be robust against non-normality and heteroscedasticity. Moreover, comparing ML with MLR estimates (while ignoring clustering) indicated small differences between standard errors, suggesting that the degree of assumption violation was small as well. Binary logistic regression was used for dichotomized versions of MVPA, total screen time, and participation in organized PA. For each dependent variable, the crude association with parental status was calculated first. This was followed by an adjusted analysis accounting for sex, age group, material affluence, having siblings, and BMI. For each continuous outcome and participation in organized PA, the interaction of parental status with sex, material affluence and having siblings was examined by means of a Wald test.

\section{Results}

Table 1 shows the characteristics of the sample. About one quarter was living in a single or a reconstituted family. The large majority $(82.8 \%)$ did not meet the $60 \mathrm{~min}$ MVPA 7 days/week with a mean of 4.1 (+2.0 SD) days/ week. The proportion not participating in organized sport was $38,2 \%$. The average total screen time was 6,1 (+4.3 SD) hours/day, with $83,6 \%$ exceeding $2 \mathrm{~h} /$ day.

In Table 2 the crude associations show that living in a single parent or a reconstituted family structure was negatively related to number of days with $60 \mathrm{~min}$ MVPA and increased the odds for less than 7 days/week and the odds for not participating in organized sport. In the adjusted model, living in a single or in a reconstituted family structure remained associated with number of days
Table 1 Baseline characteristics

\begin{tabular}{ll}
\hline Variable & Estimate \\
\hline Female sex, \% (n) & $52.8(2381)$ \\
Age category, \% (n) & \\
$\quad 11$ yrs. old & $30.2(1353)$ \\
13 yrs. old & $23.0(1030)$ \\
15 yrs. old & $19.4(869)$ \\
16yrs. old & $27.3(1223)$ \\
Material affluence, \% (n) & \\
Low & $18.3(783)$ \\
Middle & $65.5(2809)$ \\
High & $16.2(696)$ \\
Having siblings, \% (n) & $90.9(3652)$ \\
BMl, mean (SD) & $19.7(3.5)$ \\
Family structure, \% (n) & \\
Both parents & $74.1(2962)$ \\
Single parent & $15.7(628)$ \\
Reconstituted & $10.2(407)$ \\
60 min MVPA in days, mean (SD) & $4.1(2.0)$ \\
Less than 7 days 60 min MVPA, \% (n) & $82.8(3507)$ \\
Not participating in organized sport, \% (n) & $38.2(1051)$ \\
Screen-based passive entertainment (hours/day), mean (SD) & $2.1(1.6)$ \\
Gaming in hours/day, mean (SD) & $1.7(1.8)$ \\
Other screen-based activities in hours/day, mean (SD) & $2.4(2.0)$ \\
Sotal screen time in hours/day, mean (SD) & $6.1(4.3)$ \\
\hline & $83.6(2935)$ \\
\hline
\end{tabular}

with 60 min MVPA, while the odds for less than 7 days/ week and the odds for not participating in organized sport remained significantly higher for those living with a single parent.

In Table 3 the crude associations show that living in a single parent or in a reconstituted family structure was positively associated with hours/weekday screen-based passive entertainment, hours/weekday with other screen-based activities, and for those living in a reconstituted family with hours/weekday of gaming. In the adjusted model living in a single parent or in a reconstituted family structure remained positively associated with hours/weekday with other screen-based activities, and for those in reconstituted families also with hours/weekday of gaming. Other significant predictors of screen time activities were gender, age and BMI, the latter not for hours/ weekday of gaming.

Results for screen time activities in weekends were largely in line with the ones reported for weekdays (not shown in Table 3). One notable exception was that the association between screen-based passive entertainment in weekends and reconstituted families remained significant in the adjusted analyses ( $\mathrm{b}=.27$; 95\%CI: .08, .46, $p=.005)$. 
Table 2 Crude and adjusted associations of family structure with physical activity ${ }^{a}$

\begin{tabular}{|c|c|c|c|c|c|c|}
\hline & \multicolumn{2}{|c|}{60 MIN MVPA (days) } & \multicolumn{2}{|c|}{ Less than 7 days 60 MIN MVPA } & \multicolumn{2}{|c|}{ Not participating in organized PA } \\
\hline & $\mathrm{b}$ & $95 \% \mathrm{Cl}$ & Odds ratio & $95 \% \mathrm{Cl}$ & Odds ratio & $95 \% \mathrm{Cl}$ \\
\hline \multicolumn{7}{|l|}{ Crude } \\
\hline \multicolumn{7}{|l|}{ Family structure } \\
\hline Single parent & -.58 & $(-.78,-.38)$ & 1.55 & $(1.19,2.02)$ & 1.66 & $(1.31,2.10)$ \\
\hline Reconstituted & -.46 & $(-.69,-.23)$ & 1.57 & $(1.13,2.19)$ & 1.33 & $(1.03,1.72)$ \\
\hline \multicolumn{7}{|l|}{ Adjusted } \\
\hline Female sex & -.47 & $(-.60,-.34)$ & 2.20 & $(1.84,2.64)$ & 1.30 & $(1.05,1.61)$ \\
\hline \multicolumn{7}{|l|}{ Age category } \\
\hline 11 yrs. old & .89 & $(.57,1.22)$ & .55 & $(.37, .83)$ & - & - \\
\hline 13 yrs. old & .50 & $(.20, .79)$ & .97 & $(.66,1.43)$ & .32 & $(.24, .43)$ \\
\hline 15 yrs. old & .16 & $(-.11, .44)$ & .86 & $(.58,1.27)$ & .64 & $(.49, .83)$ \\
\hline \multicolumn{7}{|l|}{ Material affluence } \\
\hline Low & -.72 & $(-.93,-.50)$ & 1.76 & $(1.31,2.38)$ & 1.77 & $(1.34,2.34)$ \\
\hline Medium & -.50 & $(-.67,-.33)$ & 1.52 & $(1.22,1.90)$ & 1.19 & $(.94,1.50)$ \\
\hline Having siblings & .15 & $(-.07, .38)$ & .92 & $(.66,1.28)$ & .72 & $(.55, .94)$ \\
\hline BMl & -.04 & $(-.07,-.02)$ & 1.06 & $(1.03,1.11)$ & 1.05 & $(1.02,1.08)$ \\
\hline \multicolumn{7}{|l|}{ Family structure } \\
\hline Single parent & -.39 & $(-.58,-.20)$ & 1.36 & $(1.04,1.78)$ & 1.40 & $(1.09,1.79)$ \\
\hline Reconstituted & -.31 & $(-.53,-.08)$ & 1.36 & $(.98,1.89)$ & 1.25 & $(.96,1.63)$ \\
\hline
\end{tabular}

${ }^{a} b$ Unstandardized regression coefficient; reference category for age category $=16 \mathrm{yr}$. olds; for material affluence High; for parental status, both parents. Estimates in bold indicate statistical significance at the $p<.05$ level

Table 3 Crude and adjusted associations of family structure with weekday screen-based passive entertainment, gaming, and other screen-based activities ${ }^{a}$

\begin{tabular}{|c|c|c|c|c|c|c|}
\hline & \multicolumn{2}{|c|}{ Screen-based passive entertainment (hours/weekday) } & \multicolumn{2}{|c|}{ Gaming (hours/weekday) } & \multicolumn{2}{|c|}{ Other screen-based activities (hours/weekday) } \\
\hline & $\mathrm{b}$ & $95 \% \mathrm{Cl}$ & $\mathrm{b}$ & $95 \% \mathrm{Cl}$ & $\mathrm{b}$ & $95 \% \mathrm{Cl}$ \\
\hline \multicolumn{7}{|l|}{ Crude } \\
\hline \multicolumn{7}{|l|}{ Family structure } \\
\hline Single parent & .21 & $(.05, .38)$ & .09 & $(-.09, .27)$ & .36 & $(.16, .57)$ \\
\hline Reconstituted & .23 & $(.04, .42)$ & .22 & $(.001, .43)$ & .63 & $(.39, .86)$ \\
\hline \multicolumn{7}{|l|}{ Adjusted } \\
\hline Female sex & -.29 & $(-.40,-.17)$ & -.84 & $(-.98,-.71)$ & .17 & $(.04, .31)$ \\
\hline \multicolumn{7}{|l|}{ Age category } \\
\hline 11 yrs. old & -.46 & $(-.70,-.33)$ & -.06 & $(-.32, .20)$ & -1.37 & $(-1.60,-.13)$ \\
\hline 13 yrs. old & -.01 & $(-.22, .17)$ & .23 & $(-.03, .48)$ & -.49 & $(-.75,-.23)$ \\
\hline 15 yrs. old & .20 & $(-.04, .32)$ & .30 & $(.05, .55)$ & -.06 & $(-.30, .19)$ \\
\hline \multicolumn{7}{|l|}{ Material affluence } \\
\hline Low & .05 & $(-.15, .24)$ & .02 & $(-.20, .23)$ & -.05 & $(-.28, .18)$ \\
\hline Medium & .03 & $(-.13, .19)$ & -.05 & $(-.22, .12)$ & .03 & $(-.22, .16)$ \\
\hline Having siblings & -.17 & $(-.38, .03)$ & .05 & $(-.16, .25)$ & .02 & $(-.21, .24)$ \\
\hline $\mathrm{BMI}$ & .04 & $(.01, .05)$ & .02 & $(-.002, .05)$ & .04 & $(.02, .07)$ \\
\hline \multicolumn{7}{|l|}{ Family structure } \\
\hline Single parent & .15 & $(-.01, .31)$ & .09 & $(-.08, .26)$ & .25 & $(.06, .43)$ \\
\hline Reconstituted & 17 & $(-.01, .35)$ & .26 & $(.05, .48)$ & .44 & $(.22, .66)$ \\
\hline
\end{tabular}


In Table 4, the crude associations demonstrate a significant relationship between living in a reconstituted family and total hours/weekday of screen time as well as exceeding two hours/weekday. There was also a positive but weaker association between living with a single parent and total hours/day of screen time. In the adjusted model, gender, age and BMI were significant predictors. Controlling for covariates had a small reduction on the strength of the observed associations.

Again, the results for total and recommended screen time in weekend days were largely in line with the one reported for weekdays (not shown in Table 4), although only borderline significant associations were found in the adjusted analyses for total screen time and single parent families $(\mathrm{b}=.43,95 \% \mathrm{CI}:-.05, .91, p=.08)$, and for recommended screen time and reconstituted families $(\mathrm{OR}=1.89$; 95\%CI: .91, 3.93, $p=.09$ ).

For all but one, the interaction effects of family structure with sex, material affluence and having siblings were not statistically significant. A statistically significant interaction effect was found between family structure and material affluence for participation in organized sports only $\left(\chi^{2}[4]=13.9, p=.008\right)$. Living in a reconstituted family was associated with an increased risk for not participating in organized sports for young people who reported low $(\mathrm{OR}=3.14, p=.004)$ and high material affluence $(\mathrm{OR}=2.08, p=.01)$, but not those who reported medium material affluence $(\mathrm{OR}=.90$, $p=.57)$.

\section{Discussion}

This study sought to examine if family structure was associated with young people's MVPA, participation in organized sport and time spent on various screen activities after school. After adjusting for gender, age, material affluence, having siblings and BMI, the results showed that living in a single parent or a reconstituted family was negatively associated with days/week of $60 \mathrm{~min}$ MVPA and positively associated with hours/weekday with total screen time and hours/week with screen-based activities other than gaming and passive entertainment. Furthermore, living with a single parent increased the odds for less than 7 days/week of 60 min MVPA and for not participating in organized sport, while living in reconstituted families increased the odds for exceeding $2 \mathrm{~h} /$ weekday of total screen time and was positively associated with hours/day of gaming and with hours/weekend-days of screen-based passive entertainment.

\section{Family structure, physical activity and sport}

One key finding was that living with a single parent or in a reconstituted family was negatively associated with

Table 4 Crude and adjusted associations of family structure with total and recommended screen time ${ }^{a}$

\begin{tabular}{|c|c|c|c|c|}
\hline & \multicolumn{2}{|c|}{ Total screen time (hours/weekday) } & \multicolumn{2}{|c|}{$\begin{array}{l}\text { Screen time more than } \\
\text { two hours per weekday }\end{array}$} \\
\hline & $\mathrm{b}$ & $95 \% \mathrm{Cl}$ & Odds ratic & $95 \% \mathrm{Cl}$ \\
\hline \multicolumn{5}{|l|}{ Crude } \\
\hline \multicolumn{5}{|l|}{ Family structure } \\
\hline Single parent & .68 & $(.23,1.14)$ & 1.08 & $(.82,1.43)$ \\
\hline Reconstituted & 1.04 & $(.54,1.54)$ & 1.62 & $(1.14,2.31)$ \\
\hline \multicolumn{5}{|l|}{ Adjusted } \\
\hline Female sex & -.96 & $(-1.27,-.66)$ & .65 & $(.54, .78)$ \\
\hline \multicolumn{5}{|l|}{ Age category } \\
\hline 11 yrs. old & -1.91 & $(-2.51,-1.30)$ & .37 & $(.28, .50)$ \\
\hline 13 yrs. old & -.34 & $(-.96, .28)$ & .81 & $(.59,1.11)$ \\
\hline 15 yrs. old & .45 & $(-.11,1.02)$ & 1.11 & $(.80,1.54)$ \\
\hline \multicolumn{5}{|l|}{ Material affluence } \\
\hline Low & .05 & $(-.48, .58)$ & .90 & $(.65,1.26)$ \\
\hline High & -.05 & $(-.48, .38)$ & .95 & $(.74,1.23)$ \\
\hline Having siblings & -.07 & $(-.57, .43)$ & .85 & $(.58,1.25)$ \\
\hline BMI & .10 & $(.05, .16)$ & 1.06 & $(1.02,1.09)$ \\
\hline \multicolumn{5}{|l|}{ Family structure } \\
\hline Single parent & .50 & $(.08, .93)$ & .99 & $(.74,1.31)$ \\
\hline Reconstituted & .85 & $(.37,1.33)$ & 1.48 & $(1.02,2.14)$ \\
\hline
\end{tabular}

${ }^{a} \mathrm{~b}=$ unstandardized regression coefficient; reference category for age category $=16 \mathrm{yr}$. olds; for material affluence $=$ middle; for parental status $=$ both parents. Estimates in bold indicate statistical significance at the $p<.05$ level 
number of days/week of 60 min MVPA, with the strongest relationship observed for single parent homes. No evidence was found that the association between MVPA and family structure differed across gender, material affluence and having siblings.

Youth from single parent families had also increased odds for less than 7 days/week of $60 \mathrm{~min}$ MVPA. We have not identified other studies that have examined and documented a relationship between family structure and the physical activity recommendations. This finding should therefore be of public health interest, as it suggests that Norwegian youth living with single parents might be potentially "at increased risk", for not fulfilling the physical activity recommendations. We also found increased odds for not participating in organized sport when living with a single parent. Furthermore, there was an interesting interaction effect observed for young people living in a reconstituted family that had an increased risk for not participating in organized sports if they reported low or high material affluence. This finding suggests that complex processes are involved, and highlights the need for more in depth research on these relationships. Our results are largely in line with the findings from a study conducted in Canada [31], where young people from both single-parent and reconstituted families were less likely to participate in organized sport than those living with both their biological parents. In the Canadian study, perceived family wealth partially mediated the relationship between family structure and organized PA participation [32]. Family affluence was in our study a predictor of organized sport participation as well, suggesting that economic factors, such as costs related to sport participation, may partly explain the observed relationship.

In addition, it has been suggested that parents' time provides the combination of support and control associated with positive child outcomes [24, 35]. Qualitative studies have reported that children in single parent families experience more barriers for engaging in physical activities than children in traditional families, and receive less parental support due to lack of free time, work load, and household responsibilities [35]. In Norway, the majority of sports organized for young people is carried out by volunteers among parents [68], i.e. parents are expected to be involved in the sports clubs doing voluntary work. Lack of time may thus influence the ability to take on such responsibilities. Young people will also often be dependent on their parents for transportation to both training facilities and to competitions. Another factor that has been reported to restrict engagement in physical activities among children in non-traditional families is time to travel to visit biological parents [35]. Thus, issues related to time constraints seem possible additional explanations for the identified relationships.

\section{Family structure and screen time use}

A second key finding was that living with a single parent or in a reconstituted family was positively associated with hours/weekday of total screen time and with hours/ day of screen-based activities other than gaming and passive entertainment, with the strongest associations found for children in reconstituted families. This type of family structure also increased the odds for exceeding 2 $\mathrm{h} /$ weekday of total screen time and was positively associated with hours/day of gaming. Adjusting for covariates had only a small reduction on the strength of the associations. No evidence was found that the association between screen time use and family structure differed across gender, material affluence and having siblings.

These results contrast the findings of McMillan et al. (2015) who based on the same screen time items, found generally no significant differences between Canadian youth from traditional and non-traditional families in hours/week of total screen time, nor higher odds to exceed screen time guidelines, or to be in the highest quartile of video game or computer use [21]. A study of English youth found higher levels of sedentary activities among those in single-parents but not in step-parent families [35]. Another English study found that boys, but not girls, had higher levels of total sedentary time on weekdays and weekend, and higher levels of weekday computer use and of weekend TV viewing, when living with a single parent compared with dual-parent households [37]. Several studies [6, 20, 21, 35, 37, 39, 69] have not found an association between family structure and $\mathrm{TV}$ viewing. Our results of screen-based passive entertainment (including watching TV) are largely in line with these findings with the exception of a significant and positive association between reconstituted families and screen-based passive entertainment in weekends.

The reasons for the observed relationships in the current study are likely multifaceted. Less involvement in physical activity and sport for these adolescents may be one factor that could result in sedentary pursuits [35]. A family level construct that has been linked to positive health behaviors is family cohesion, defined as the "emotional bonding that family members have toward one another" [70]. Some studies indicate that stepparents are less committed to their non-biological children [22, 71], it has also been suggested that reconstituted families need time to establish a new family structure and reorganize [72]. The stress and instability perspective suggests that changes in family structure may disrupt the equilibrium of family environments for many years, with elevated stress that interrupts effective parenting behaviors [73, 74]. These aspects, together with the time factor, may result in less support and involvement in parenting, as well as challenges when enforcing screen time limitations on youth that, in sum, 
influence screen-based behaviors. The findings in the current study suggest the need for a more in-depth assessment of the relationship between family structures and screen-based behaviors that can uncover the processes and shed light of the mechanisms through which family structures may influence the behaviors.

\section{Strengths and limitations}

A strength of the study was the large sample of Norwegian youth with sufficient statistical power to examine main and interaction effects related to family structure. The study also controlled for several potential covariates. Another strength was the use of well-established measures developed by expert teams within the international HBSC study, a study that has comprehensive methodological data collection procedures. The study has several limitations. A known limitation is that all data were self-reported, which is known to have recall and reporting bias. However, most of the items have been documented to have satisfying validity and reliability [45].

Another limitation was that the proportion reporting other types of family structures was in the current study insufficient to examine the relationships with less common, but potentially important family types. We also lacked information on how long the participants had been living in their current family structure, limiting our ability to study the processes involved. Another limitation is the use of cross-sectional data, which does not allow for causal inference. In addition, the issue with omitted variable bias makes it difficult to propose any causality. The factors that affect the type of family structure parents choose may also affect parental resources, as well as the physical activity and sedentary behaviors of the children. If a larger set of parental factors, such as educational level, could have been included as covariates, more of the potential unobserved sources of the observed association could have been accounted for.

\section{Implications}

The finding that family structure was a correlate for MVPA, sport participation and screen-time activities among Norwegian youth, should be of interest for public health researchers and practitioners as the proportion of young people living in family structures consisting of others than both their biological mother and father, is substantial. The study suggests that young people living with a single parent are "at risk" for not meeting the MVPA-recommendations and for not participating in organized sport. This is an important finding as sport can contribute to a significant amount of young peoples' total physical activity, in particular vigorous activity [42, $43,75]$. In a Norwegian setting, it could be worthwhile to address structural and organizational barriers that single parents may experience. Those living in reconstituted families were "at-risk" for exceeding $2 \mathrm{~h}$ of screen-time use, with higher levels of both gaming and screen-based activities other than passive entertainment. So far, few studies have identified correlates of sedentary time with an attention to the after-school period, and those that exist have mainly focused on TV viewing [8, 44]. The fact that computer use has sharply increased [4], that it has been limited effect of interventions to reduce sedentary time [8], and that the relationship with many of the determinants of sedentary behavior remain inconsistent [76], underline the importance of more studies on correlates. Most of the research on family structure has typically assessed single versus dual families. The identified relationships between living in a reconstituted family and lower levels of physical activity as well as higher levels of screen time use compared to two parent families are interesting and warrant further investigation of the mechanisms involved. A recent nationally representative study of Norwegian 8 year olds found that objectively measured overweight was more prevalent among children of divorced parents [77]. The identified relationships between family structure and physical activity and sedentary activities could thus be a pathway to overweight. Longitudinal and also studies with a qualitative design could shed light on the processes and the complexity of the examined associations.

As the majority of the participants in this study did not meet the guidelines for PA and SB regardless of family structure, universal interventions are warranted to increase levels of PA and reduce levels of SB. However, the study findings suggest that family structure may be a factor to account for in the development of interventions, for example by examining ways to intensify interventions for single and reconstituted families (i.e. proportionate universalism [78]). It would also be of interest to examine whether family structure acts as a moderator for intervention effects.

\section{Conclusion}

The study demonstrated that family structure was a correlate of physical activity, sport participation and screen time behaviors among Norwegian youth. These findings suggest that family structure could be an important factor to take into account in the development of interventions and programs. More research on this topic is warranted.

\footnotetext{
Abbreviations

BMI: Body mass index; Cl: Confidence interval; EU: European Union; FAS III: Family affluence scale III; FIML: Full information maximum likelihood; HBSC: Health Behaviour in School- aged Children; MVPA: Moderate to vigorous physical activity; OR: Odds ratio; PA: Physical activity; SD: Standard deviation; WHO: World Health Organization
}

\section{Acknowledgements}

The Health Behavior in School-Aged Children (HBSC) is an international study carried out in collaboration with World Health Organization/Europe WHO/ EURO. We wish to acknowledge the International coordinator of the 2013/ 
2014 survey, Candace Currie and her team at the University of Edinburgh, Scotland, and the International Data Centre Manager, Oddrun Samdal and her team at the University of Bergen, Norway. We also wish to thank all the participating students, staff and schools that took part in the Norwegian survey.

\section{Funding}

The Norwegian part of the HBSC-study was conducted of the University of Bergen. The data collection was partly funded by the Norwegian Directorate of Health. This funding body was not involved in the design of this study or in any parts of the manuscript development.

\section{Availability of data and materials}

The University of Bergen is the data-bank manager for the international HBSC study. Please contact corresponding author for data requests.

\section{Authors' contributions}

First author, $\mathrm{AL}$, and last author, EH, conceived the idea, led the development of the manuscript interpreted the data and drafted the paper ORFS analyzed the data, interpreted the data and contributed to the writing. BW contributed to the writing and OS administered the study, secured funding and contributed to the writing. All authors have read and approved the final manuscript.

\section{Ethics approval and consent to participate}

The Norwegian Western Regional Ethical Committee (REK) approved the study and the use of informed passive consent under the condition that a detailed information letter was given both in paper form and electronically to parents or custodians for all participants below the age of 16. Those that did not want their child to participate had to sign and return a form to the teacher. Approval of the child's participation was assumed if the form was not returned.

\section{Consent for publication}

Not applicable.

\section{Competing interests}

The authors declare that they have no competing interests.

\section{Publisher's Note}

Springer Nature remains neutral with regard to jurisdictional claims in published maps and institutional affiliations.

\section{Author details}

NLA University College, Bergen, Pb 74 Sandviken, 5812 Bergen, Norway. 2Department of Health Promotion, Norwegian Institute of Public Health, Bergen, Zander Kaaesgate 7, 5015 Bergen, Norway. ${ }^{3}$ Department of Health Promotion and Development, University of Bergen, Christiesgate 13, 5020 Bergen, Norway.

\section{Received: 26 May 2018 Accepted: 2 April 2019}

\section{Published online: 25 April 2019}

\section{References}

1. Janssen I, LeBlanc AG. Systematic review of the health benefits of physical activity and fitness in school-aged children and youth. Int J Behav Nutr Phys Act. 2010;7(1):1.

2. Jiménez-Pavón D, Kelly J, Reilly JJ. Associations between objectively measured habitual physical activity and adiposity in children and adolescents: systematic review. Int J Pediatr Obes. 2010;5(1):3-18.

3. Eime RM, et al. A systematic review of the psychological and social benefits of participation in sport for children and adolescents: informing development of a conceptual model of health through sport. Int J Behav Nutr Phys Act. 2013;10(1):1

4. World Health Organization. Adolescent obesity and related behaviours: trends and inequalities in the WHO European Region, 2002-2014. Geneva: World Health Organization; 2017.

5. Must A, Tybor D. Physical activity and sedentary behavior: a review of longitudinal studies of weight and adiposity in youth. Int J Obes. 2005;29: S84-96.
6. Salmon J, et al. Association of family environment with children's television viewing and with low level of physical activity. Obesity. 2005;13(11):1939-51.

7. Owen $\mathrm{N}$, et al. Too much sitting: the population-health science of sedentary behavior. Exerc Sport Sci Rev. 2010;38(3):105.

8. Salmon J, et al. Health risks, correlates, and interventions to reduce sedentary behavior in young people. Am J Prev Med. 2011:41(2):197-206.

9. Russ SA, et al. Associations between media use and health in US children. Acad Pediatr. 2009;9(5):300-6.

10. Committee, P.A.G.A. Physical activity guidelines for Americans. Washington, DC: US Department of Health and Human Services; 2008. p. 15-34.

11. Bar-on ME. The effects of television on child health: implications and recommendations. Arch Dis Child. 2000;83(4):289-92.

12. Tremblay MS, et al. Canadian sedentary behaviour guidelines for children and youth. Appl Physiol Nutr Metab. 2011;36(1):59-64.

13. Trost, S., Discussion paper for the development of recommendations for children's and youth's participation in health promoting physical activity. 2005.

14. Sallis JF, Owen N, Fotheringham MJ. Behavioral epidemiology: a systematic framework to classify phases of research on health promotion and disease prevention. Ann Behav Med. 2000;22(4):294-8.

15. Biddle SJ, et al. Correlates of physical activity in youth: a review of quantitative systematic reviews. Int Rev Sport Exerc Psychol. 2011:4(1):25-49.

16. Gustafson SL, Rhodes RE. Parental correlates of physical activity in children and early adolescents. Sports Med. 2006;36(1):79-97.

17. Biddle SJ, Petrolini I, Pearson N. Interventions designed to reduce sedentary behaviours in young people: a review of reviews. Br J Sports Med. 2013. https://doi.org/10.1136/bjsports-2013-093078.

18. Amato PR. The consequences of divorce for adults and children: an update. Društvena istraživanja-Časopis za opća društvena pitanja. 2014;1:5-24.

19. Eurostat. Employment, Social Affairs \& Inclusion. In: Employment, Social Affairs \& Inclusion. Luxembourg: Publications Office of the European Union; 2015.

20. Bagley S, Salmon J, Crawford D. Family structure and children's television viewing and physical activity. Med Sci Sports Exerc. 2006:38(5):910-8.

21. McMillan R, Mclsaac M, Janssen I. Family structure as a predictor of screen time among youth. PeerJ. 2015;3:e1048.

22. Wang L, Qi J. Association between family structure and physical activity of Chinese adolescents. Biomed Res Int. 2016:1:1-7.

23. Ram B, Hou F. Changes in family structure and child outcomes: roles of economic and familial resources. Policy Stud J. 2003;31(3):309-30.

24. Thomson E, McLanahan SS. Reflections on "family structure and child wellbeing: economic resources vs. parental socialization". Social Forces. 2012; 91(1):45-53.

25. McLanahan S, Percheski C. Family structure and the reproduction of inequalities. Annu Rev Sociol. 2008;34:257-76.

26. Tillman $\mathrm{KH}$. Family structure pathways and academic disadvantage among adolescents in stepfamilies. Sociol Inq. 2007:77(3):383-424.

27. Thomson E, Hanson TL, McLanahan SS. Family structure and child wellbeing: economic resources vs. parental behaviors. Social Forces. 1994;73(1): 221-42.

28. Ferreira I, et al. Environmental correlates of physical activity in youth-a review and update. Obes Rev. 2007;8(2):129-54.

29. Sallis JF, et al. Correlates of vigorous physical activity for children in grades through 12: comparing parent-reported and objectively measured physical activity. Pediatr Exerc Sci. 2002;14(1):30-44.

30. Theodorakis Y, Papaioannou A, Karastogianidou K. Relations between family structure and students' health-related attitudes and behaviors. Psychol Rep. 2004;95(3):851-8.

31. McMillan R, Mclsaac M, Janssen I. Family structure as a correlate of organized sport participation among youth. PLoS One. 2016;11(2):e0147403.

32. Toftegaard-Støckel J, et al. Parental, socio and cultural factors associated with adolescents' sports participation in four Danish municipalities. Scand J Med Sci Sports. 2011;21(4):606-11.

33. Eime RM, et al. Does sports club participation contribute to health-related quality of life. Med Sci Sports Exerc. 2010;42(5):1022-8.

34. Musick K, Meier A. Are both parents always better than one? Parental conflict and young adult well-being. Soc Sci Res. 2010;39(5):814-30

35. Quarmby T, Dagkas S, Bridge M. Associations between children's physical activities, sedentary behaviours and family structure: a sequential mixed methods approach. Health Educ Res. 2010;26(1):63-76.

36. Quarmby T, Dagkas S. Children's engagement in leisure time physical activity: exploring family structure as a determinant. Leis Stud. 2010;29(1): 53-66. 
37. Gorely T, et al. Family circumstance, sedentary behaviour and physical activity in adolescents living in England: project STIL. Int J Behav Nutr Phys Act. 2009;6(1):33

38. Gorely T, Marshall SJ, Biddle SJ. Couch kids: correlates of television viewing among youth. Int J Behav Med. 2004;11(3):152-63.

39. Hesketh K, Crawford D, Salmon J. Children's television viewing and objectively measured physical activity: associations with family circumstance. Int J Behav Nutr Phys Act. 2006;3(1):36.

40. Kjønniksen L, Anderssen N, Wold B. Organized youth sport as a predictor of physical activity in adulthood. Scand J Med Sci Sports. 2009;19(5):646-54

41. Mahoney, J.L., A.L. Harris, and J.S. Eccles, Organized Activity Participation, Positive Youth Development, and the Over-Scheduling Hypothesis. Social Policy Report. Volume 20, Number 4. Society for Research in Child Development, 2006.

42. Vella SA, et al. Sports participation and parent-reported health-related quality of life in children: longitudinal associations. J Pediatr. 2014;164(6): 1469-74.

43. Wickel EE, Eisenmann JC. Contribution of youth sport to total daily physical activity among 6-to 12-yr-old boys. Med Sci Sports Exerc. 2007;39(9):1493500 .

44. Arundell $L$, et al. The correlates of after-school sedentary behavior among children aged 5-18 years: a systematic review. BMC Public Health. 2016; 16(1):58.

45. Currie C. In: Currie C, et al., editors. Health Behaviour in School-aged Children (HBSC) Study Protocol: Background, Methodology and Mandatory items for the 2013/14 Survey. St. Andrews: Child and Adolescent Health Research Unit (CAHRU); 2014.

46. Biddle SJ, et al. An assessment of self-reported physical activity instruments in young people for population surveillance: project ALPHA. Int J Behav Nutr Phys Act. 2011;8(1):1.

47. Vuori M, et al. The stability of physical activity survey items in the HBSC study. Liik Tiede. 2005:42(6):39-46.

48. Ridgers ND, et al. Validity of a brief self-report instrument for assessing compliance with physical activity guidelines amongst adolescents. J Sci Med Sport. 2012;15(2):136-41.

49. World Health Organization. Global recommendations on physical activity for health. 2010. (2015).

50. Bosakova $\mathrm{L}$, et al. Test-retest reliability of the scale of participation in organized activities among adolescents in the Czech Republic and Slovakia. Int J Public Health. 2016;61(3):329-36.

51. Vereecken CA, et al. Television viewing behaviour and associations with food habits in different countries. Publ Health Nutr-Cab Int. 2006:9(2):244.

52. Liu Y, et al. Test-retest reliability of selected items of health behaviour in school-aged children (HBSC) survey questionnaire in Beijing, China. BMC Med Res Methodol. 2010;10(1):1.

53. Rey-López JP, et al. Sedentary patterns and media availability in European adolescents: the HELENA study. Prev Med. 2010;51(1):50-5.

54. Schmitz KH, et al. Reliability and validity of a brief questionnaire to assess television viewing and computer use by middle school children. J Sch Health. 2004;74(9):370-7

55. Melkevik O, et al. Are associations between electronic media use and BMI different across levels of physical activity? BMC Public Health. 2015;15(1):1.

56. Uijtdewilligen $\mathrm{L}$, et al. Determinants of physical activity and sedentary behaviour in young people: a review and quality synthesis of prospective studies. Br J Sports Med. 2011:45(11):896-905.

57. Kracht CL, Sisson SB. Sibling influence on children's objectively measured physical activity: a meta-analysis and systematic review. BMJ Open Sport Exerc Med. 2018;4:e000405. https://doi.org/10.1136/bmjsem-2018-000405.

58. McVeigh J, Norris S, Wet TD. The relationship between socio-economic status and physical activity patterns in South African children. Acta Paediatr. 2004;93(7):982-8

59. Sallis JF, Prochaska JJ, Taylor WC. A review of correlates of physical activity of children and adolescents. Med Sci Sports Exerc. 2000;32(5):963-75.

60. Hartley JE, Levin $\mathrm{K}$, Currie C. A new version of the HBSC family affluence scale-FAS III: Scottish qualitative findings from the international FAS development study. Child Indic Res. 2016;9(1):233-45.

61. Cole TJ, et al. Establishing a standard definition for child overweight and obesity worldwide: international survey. Bmj. 2000:320(7244):1240.

62. Brener ND, McManus T, Galuska DA, Lowry R, Wechsler $H$. Reliability and validity of self-reported height and weight among high school students. J Adolesc Health. 2003;32:281-7.
63. Strauss RS. Comparison of measured and self-reported weight and height in a cross-sectional sample of young adolescents. Int J Obes Relat Metab Disord. 1999;23:904-8

64. Wang Z, Patterson CM, Hills AP. A comparison of self-reported and measured height, weight and BMI in Australian adolescents. Aust N Z J Public Health. 2002;26:473-8.

65. Goodman E, Hinden BR, Khandelwal S. Accuracy of teen and parental reports of obesity and body mass index. Pediatrics. 2000;106:52-8.

66. Spencer EA, Appleby PN, Davey GK, Key TJ. Validity of self-reported height and weight in 4808 EPIC-Oxford participants. Public Health Nutr. 2002;5: $561-5$.

67. Preston SH, Fishman E, Stokes A. Effects of categorization and self-report bias on estimates of the association between obesity and mortality. Ann Epidemiol. 2015;25:907-11.e2.

68. Ibsen B, Seippel $\varnothing$. Voluntary organized sport in Denmark and Norway. Sport Soc. 2010;13(4):593-608.

69. Lindquist $\mathrm{CH}$, Reynolds KD, Goran MI. Sociocultural determinants of physical activity among children. Prev Med. 1999;29(4):305-12.

70. Olson D, Gorall D. Circumplex model of marital and family system. In: Walsh F, editor. Normal Family Processes. New York: Guilford; 2003.

71. Manning WD, Brown S. Children's economic well-being in married and cohabiting parent families. J Marriage Fam. 2006:68(2):345-62.

72. Breivik K, Olweus D. Adolescent's adjustment in four post-divorce family structures: single mother, stepfather, joint physical custody and single father families. J Divorce Remarriage. 2006;44(3-4):99-124.

73. Sweeney MM. Remarriage and stepfamilies: strategic sites for family scholarship in the 21st century. J Marriage Fam. 2010;72(3):667-84.

74. Hetherington EM, Kelly J. For better or for worse: Divorce reconsidered. New York: WW Norton \& Company; 2003.

75. Wold B, Duda JL, Balaguer I, ORF Smith, Ommundsen Y, Hall HK, Samdal O, et al. Comparing self-reported leisure-time physical activity, subjective health, and life satisfaction among youth soccer players and adolescents in a reference sample. Int J Sport Exerc Psychol. 2013:11(4):328-40.

76. Stierlin AS, et al. A systematic review of determinants of sedentary behaviour in youth: a DEDIPAC-study. Int J Behav Nutr Phys Act. 2015;12(1): 133.

77. Biehl A, et al. Parental marital status and childhood overweight and obesity in Norway: a nationally representative cross-sectional study. BMJ Open. 2014:4(6):e004502.

78. Marmot M, et al. Fair society, healthy lives. The Marmot Review. London: Institute of Health Equity; 2010.

Ready to submit your research? Choose BMC and benefit from:

- fast, convenient online submission

- thorough peer review by experienced researchers in your field

- rapid publication on acceptance

- support for research data, including large and complex data types

- gold Open Access which fosters wider collaboration and increased citations

- maximum visibility for your research: over $100 \mathrm{M}$ website views per year

At $\mathrm{BMC}$, research is always in progress.

Learn more biomedcentral.com/submissions 